\title{
A unified approach to blending of constant and varying parametric surfaces with curvature continuity
}

\author{
X. Y. You \\ Department of Creative Technology \\ Faculty of Science and Technology \\ Bournemouth University \\ Poole, BH12 5BB \\ United Kingdom \\ E-mail: xyou@bournemouth.ac.uk
}

$$
\text { F. Tian }
$$

Department of Creative Technology

Faculty of Science and Technology

Bournemouth University

Poole, BH12 5BB

United Kingdom

E-mail: ftian@bournemouth.ac.uk

\author{
W. Tang \\ Department of Creative Technology \\ Faculty of Science and Technology \\ Bournemouth University \\ Poole, BH12 5BB \\ United Kingdom \\ E-mail: wtang@bournemouth.ac.uk
}

\begin{abstract}
In this paper, we develop a new approach to blending of constant and varying parametric surfaces with curvature continuity. We propose a new mathematical model consisting of a vector-valued sixth-order partial differential equation (PDE) and time-dependent blending boundary constraints, and develop an approximate analytical solution of the mathematical model. The good accuracy and high computational efficiency are demonstrated by comparing the new approximate analytical solution with the corresponding accurate closed form solution. We also investigate the influence of the second partial derivatives on the continuity at trimlines, and apply the new approximate analytical solution in blending of constant and varying parametric surfaces with curvature continuity.
\end{abstract}

Keywords Surface blending - Constant and varying parametric surfaces - Curvature continuity. Sixth-order partial differential equations $\cdot$ Approximate analytical solution

\section{Introduction}

Surfaces used in geometric modeling can be divided into explicit, implicit, and parametric surfaces. They can be mathematically represented with $z=f(x, y), f(x, y, z)=0$, and $x=x(u, v)$, $y=y(u, v)$, and $z=z(u, v)$, respectively. Accordingly, surface blending can be grouped into explicit blending, implicit blending [1], and parametric blending $[2,3]$. Since parametric surfaces are most popular, this paper will focus on blending of constant and varying parametric surfaces with curvature continuity.

For blending of parametric surfaces, a comprehensive literature survey has been made in [4]. Although various blending methods have been developed, all these methods can deal with constant surfaces only which do not change their positions and shapes with time. By introducing a vector-valued dynamic fourthorder partial differentia equation (PDE), a new blending method has been proposed in [5] to create blending of varying parametric surfaces with tangential continuity. This paper will introduce a vector-valued sixth-order partial differentia equation to integrate

Permission to make digital or hard copies of all or part of this work for personal or classroom use is granted without fee provided that copies are not made or distributed for profit or commercial advantage and that copies bear this notice and the full citation on the first page. Copyrights for components of this work owned by others than ACM must be honored. Abstracting with credit is permitted. To copy otherwise, or republish, to post on servers or to redistribute to lists, requires prior specific permission and/or a fee. Request permissions from Permissions@acm.org. CGI 2018, June 11-14, 2018, Bintan Island, Indonesia

(C) 2018 Association for Computing Machinery. ACM ISBN 978-1-4503-6401-0/18/06 ..\$15.00 https://doi.org/10.1145/3208159.32081720 both constant and varying parametric surface blending with curvature continuity.

In what follows, we first briefly review the related work in Section 2. Next, we propose the mathematical model of constant and varying parametric surface blending with curvature continuity which consists of a vector-valued sixth-order partial differential equation and blending boundary constraints, and develop an approximate analytical solution of the mathematical model in Section 3. After that, we investigate the implementation of the developed approach, compare the obtained approximate analytical solution with the corresponding accurate closed form solution, discuss the effects of second partial derivatives on the continuity at trimlines, and examine blending of constant parametric surfaces with curvature continuity in Section 4 . Finally, we conclude the work given in this paper in Section 5 .

\section{Related work}

Various blending methods of time-independent parametric surfaces have been developed. According to the survey paper [4], these surface blending methods can be divided into: rolling-ballbased blends, spine-based blends, trimline-based blends, blends based on polyhedral methods and other methods including a cyclide solution, PDE-based blends, and Fourier-based blends. It was felt in the paper that the PDE-based blending method was a powerful means of surface generation.

Surface blending using a rolling ball is the most popular. It was introduced in [6]. With this method, a blending surface is created by rolling a ball along two primary surfaces. Various rolling-ball blending methods can be divided into constant-radius and variable-radius ones. Constant-radius rolling-ball blending methods were investigated in [6-9], and variable-radius rollingball blending methods were examined in [10-12].

Cyclides were used to create some simple blending surfaces in $[13,14]$. A Fourier-based blending method was proposed in [15]. And a symbolic approach was presented in [16] for the computation of blending surfaces.

Rolling-ball methods can create circular shapes of blending surfaces only. In general situations, the shapes of blending surfaces are noncircular. This problem can be tackled by other blending methods such as N-sided hole filling [17-22], vertex blending using S-patches [23, 24], branching blends with Pythagorean normal surfaces [25], or generating arbitrary shapes of blending surfaces with the following PDE-based methods.

Partial differential equation-based methods treat surface blending as a boundary value problem. The solution to a vectorvalued partial differential equation subjected to the constraints of boundary curves and the first, second and higher order partial derivatives at trimlines represents a blending surface. Since surface blending with tangent and curvature continuity is most frequently met in engineering and tangent continuity has been 
investigated in [5], this paper examines constant and varying parametric surface blending with curvature continuity.

PDE-based surface blending was pioneered in [26]. The main problem with PDE-based surface blending is how to solve partial differential equations. In order to raise the capability of PDEbased surface blending, various numerical methods such as the finite element method [27, 28] are developed. These numerical methods have the disadvantages of: 1) discrete boundary representations unsuitable for the requirement of good continuity, 2) heavy computational cost causing slow response, and 3) specific knowledge and skills of the numerical methods to carry out the numerical calculations. In order to overcome the disadvantages of numerical methods, some approximate analytical methods have been proposed in [29, 30].

Most partial differential equation-based methods and all other surface blending approaches only deal with constant surface blending where primary surfaces do not move and change their shapes. The work given in [5] initiated the research into surface blending of dynamic varying parametric surfaces with tangential continuity. In the paper, a vector-valued dynamic fourth-order partial differential equation is used to develop a new approach which tackles blending of varying parametric surfaces with tangential continuity [5]. This paper changes the vector-valued dynamic fourth-order partial differential equation into a vectorvalued sixth-order partial differential equation, drops the time variable, and develops an accurate and efficiency approximate analytical solution to extend its capacity to blending of constant and varying parametric surface with curvature continuity.

\section{Mathematical model and approximate} analytical solution

Assuming two varying parametric surfaces to be smoothly blended together are $\mathbf{S}_{1}(u, v, t)$ and $\mathbf{S}_{2}(u, v, t)$ with $\mathbf{S}_{1}(u, v, t)$ to be blended at the position $u=u_{1}$ and $\mathbf{S}_{2}(u, v, t)$ to be blended at the position $u=u_{2}$. The position function and the first and second partial derivatives with respect to the parametric variable $u$ at the position $u=u_{1}$ are: $\mathbf{C}_{1}(v, t)=\mathbf{S}_{1}\left(u_{1}, v, t\right), \mathbf{C}_{2}(v, t)=\partial \mathbf{S}_{1}\left(u_{1}, v, t\right) / \partial u$ and $\mathbf{C}_{3}(v, t)=\partial^{2} \mathbf{S}_{1}\left(u_{1}, v, t\right) / \partial u^{2}$, respectively. And the position function and the first and second derivatives at the position $u=u_{2}$ are: $\quad \mathbf{C}_{4}(v, t)=\mathbf{S}_{2}\left(u_{2}, v, t\right) \quad, \quad \mathbf{C}_{5}(v, t)=\partial \mathbf{S}_{2}\left(u_{2}, v, t\right) / \partial u \quad$ and $\mathbf{C}_{6}(v, t)=\partial^{2} \mathbf{S}_{2}\left(u_{2}, v, t\right) / \partial u^{2}$, respectively. If the blending surface $\mathbf{S}(u, v, t)$ connects two varying parametric surfaces at the positions $u=u_{1}$ and $u=u_{2}$ smoothly, it must satisfy the following blending boundary constraints.

$$
\begin{aligned}
& u=0 \quad \partial^{n} \mathbf{S}(u, v, t) / \partial u^{n}=\mathbf{C}_{n+1}(v, t) \\
& u=1 \quad \partial^{n} \mathbf{S}(u, v, t) / \partial u^{n}=\mathbf{C}_{n+4}(v, t) \\
& (n=0,1,2)
\end{aligned}
$$

where $\quad \partial^{0} \mathbf{S}(u, v, t) / \partial u^{0}=\mathbf{S}(u, v, t) \quad, \quad \mathbf{S}(u, v, t) \quad$ has three components $S_{x}(u, v, t), S_{y}(u, v, t)$, and $S_{z}(u, v, t)$, and $\mathbf{C}_{n}(v, t)$ $(n=1,2, \cdots, 6)$ also have three components $C_{x n}(v, t), C_{y n}(v, t)$, and $C_{z n}(v, t)$.

The solution of a vector-valued sixth order partial differential equation contains 6 unknown constants. They can be used to satisfy the 6 constraints of the position functions and the first and second partial derivatives in blending boundary constraints (1).
Therefore, we choose the following vector-valued sixth order partial differential equation

$$
\left(\frac{\partial^{6}}{\partial u^{6}}+\frac{\partial^{6}}{\partial u^{4} \partial v^{2}}+\frac{\partial^{6}}{\partial u^{2} \partial v^{4}}+\frac{\partial^{6}}{\partial v^{6}}\right) \mathbf{S}(u, v, t)=0
$$

for surface blending with curvature continuity.

It is difficult to directly solve the mathematical model (2) subjected to the blending boundary constraints (1). In order to simplify the solution, we introduce $\mathbf{C}_{n}(v, t)=\mathbf{C}_{n}^{(1)}+\mathbf{C}_{n}^{(2)}(t)+\mathbf{C}_{n}^{(3)}(v)+\mathbf{C}_{n}^{(4)}(v, t) \quad(n=1,2,3, \cdots, 6)$ into (1) and substitute $\mathbf{S}(u, v, t)=\mathbf{S}^{(1)}(u)+\mathbf{S}^{(2)}(u, t)+\mathbf{S}^{(3)}(u, v)+\mathbf{S}^{(4)}(u, v, t)$ into (2), and change the mathematical model into the four sub mathematical models which consist of the sixth-order partial differential equations below

$$
\begin{aligned}
& \left(\frac{\partial^{6}}{\partial u^{6}}+\frac{\partial^{6}}{\partial u^{4} \partial v^{2}}+\frac{\partial^{6}}{\partial u^{2} \partial v^{4}}+\frac{\partial^{6}}{\partial v^{6}}\right) \mathbf{S}^{(i)}=0 \\
& (i=1,2,3,4)
\end{aligned}
$$

and the following blending boundary constraints

$$
\begin{aligned}
& u=0 \quad \partial^{n} \mathbf{S}^{(i)} / \partial u^{n}=\mathbf{C}_{n+1}^{(i)} \\
& u=1 \quad \partial^{n} \mathbf{S}^{(i)} / \partial u^{n}=\mathbf{C}_{n+4}^{(i)} \\
& (n=0,1,2 ; i=1,2,3,4)
\end{aligned}
$$

where $\quad \mathbf{S}^{(1)}=\mathbf{S}^{(1)}(u) \quad, \quad \mathbf{S}^{(2)}=\mathbf{S}^{(2)}(u, t) \quad, \quad \mathbf{S}^{(3)}=\mathbf{S}^{(3)}(u, v) \quad$, $\mathbf{S}^{(4)}=\mathbf{S}^{(4)}(u, v, t), \quad \mathbf{C}_{n}^{(1)}=\mathbf{D}_{n}, \quad \mathbf{C}_{n}^{(2)}=\mathbf{C}_{n}^{(2)}(t), \quad \mathbf{C}_{n}^{(3)}=\mathbf{C}_{n}^{(3)}(v)$, $\mathbf{C}_{n}^{(4)}=\mathbf{C}_{n}^{(4)}(v, t)$, and $\mathbf{D}_{n}(n=1,2, \cdots, 6)$ are the vector-valued constants.

When $i=1$, the boundary functions $\mathbf{C}_{n}^{(1)}=\mathbf{D}_{n}(n=1,2, \cdots, 6)$ are constants, and the closed form solution can be taken to be the form of

$$
\mathbf{S}^{(1)}(u)=\sum_{n=0}^{5} \mathbf{a}_{n}^{(1)} u^{n}
$$

The above vector-valued function has exactly satisfied the partial differential equation (3). Therefore, we substitute it into the corresponding blending boundary constraints (4) to determine the unknown vector-valued constants $\mathbf{a}_{n}^{(1)}$. After substituting the determined vector-valued constants $\mathbf{a}_{n}^{(1)}$ back to Eq. (5), $\mathbf{S}^{(1)}(u)$ is found to be

$$
\mathbf{S}^{(1)}(u)=\sum_{n=1}^{6} g_{n}(u) \mathbf{D}_{n}
$$

When $i=2$, the boundary functions $\mathbf{C}_{n}^{(2)}=\mathbf{C}_{n}^{(2)}(t)$ $n=1,2, \cdots, 6)$ are the functions of the time variable $t$, and the closed form solution can be written in the form below

$$
\mathbf{S}^{(2)}(u, t)=\sum_{n=1}^{6} g_{n}(u) \mathbf{C}_{n}^{(2)}(t)
$$

When $i=3$, the boundary functions $\mathbf{C}_{n}^{(3)}=\mathbf{C}_{n}^{(3)}(v)$ $n=1,2, \cdots, 6)$ are the functions of the parametric variable $v$, and the approximate analytical solution has the form below

$$
\begin{aligned}
& \mathbf{S}^{(3)}(u, v)=\sum_{n=1}^{6}\left\{\sum_{l=1}^{6} \mathbf{d}_{n, l} g_{l}(u)-\sum_{m=1}^{M} \mathbf{b}_{n m}\right. \\
& \left.\left[m \pi f_{m}(u)-\sin m \pi u\right]\right\} \mathbf{C}_{n}^{(3)}(v)
\end{aligned}
$$

where $\mathbf{d}_{n, m}(n, m=1,2, \cdots, 6)$ have three components $d_{q n, m}($ $q=x, y, z)$ with $d_{q n, m}=1$ for $n=m$ and $d_{q n, m}=0$ for $n \neq m$, $g_{l}(u) \quad(l=1,2, \cdots, 6)$ are the same as those in Eq. (6), $f_{m}(u)=g_{2}(u)+(-1)^{m} g_{5}(u)$, and $\mathbf{b}_{n m}$ are determined by 


$$
\begin{aligned}
& \sum_{m=1}^{M} \sum_{i=0}^{I} \sum_{j=0}^{J} \mathbf{b}_{n m} \boldsymbol{\alpha}_{n m}\left(u_{i}, v_{j}\right) \boldsymbol{\alpha}_{n q}\left(u_{i}, v_{j}\right) \\
& =\sum_{i=0}^{I} \sum_{j=0}^{J} \boldsymbol{\varphi}_{n}\left(u_{i}, v_{j}\right) \boldsymbol{\alpha}_{n q}\left(u_{i}, v_{j}\right) \\
& (n=1,2, \cdots, 6 ; q=1,2,3,4, \cdots, M)
\end{aligned}
$$

In the above equation, $\boldsymbol{\alpha}_{n m}\left(u_{i}, v_{j}\right)$ and $\boldsymbol{\alpha}_{n q}\left(u_{i}, v_{j}\right)$ are obtained from

$$
\begin{aligned}
& \boldsymbol{\alpha}_{n m}\left(u_{i}, v_{j}\right)=m^{6} \pi^{6} \mathbf{C}_{n}^{(3)}\left(v_{j}\right) \sin \left(m \pi u_{i}\right)+\left[m \pi f_{m}^{(4)}\left(u_{i}\right)\right. \\
& \left.-m^{4} \pi^{4} \sin \left(m \pi u_{i}\right)\right] \partial^{2} \mathbf{C}_{n}^{(3)}\left(v_{j}\right) / \partial v^{2}+\left[m \pi f_{m}^{(2)}\left(u_{i}\right)+m^{2} \pi^{2}\right. \\
& \left.\sin \left(m \pi u_{i}\right)\right] \partial^{4} \mathbf{C}_{n}^{(3)}\left(v_{j}\right) / \partial v^{4}+\left[m \pi f_{m}^{(0)}\left(u_{i}\right)-\sin (m \pi u)\right] \\
& \partial^{6} \mathbf{C}_{n}^{(3)}\left(v_{j}\right) / \partial v^{6} \\
& (n=1,2, \cdots, 6 ; m=1,2, \cdots, M)
\end{aligned}
$$

and $\boldsymbol{\varphi}_{n}\left(u_{i}, v_{j}\right)$ is determined by the following equation

$$
\begin{aligned}
& \boldsymbol{\varphi}_{n}\left(u_{i}, v_{j}\right)=\sum_{l=1}^{6} \mathbf{d}_{n, l}\left[g_{l}^{(6)}\left(u_{i}\right) \mathbf{C}_{n}^{(3)}\left(v_{j}\right)+g_{l}^{(4)}\left(u_{i}\right)\right. \\
& \partial^{2} \mathbf{C}_{n}^{(3)}\left(v_{j}\right) / \partial v^{2}+g_{l}^{(2)}\left(u_{i}\right) \partial^{4} \mathbf{C}_{n}^{(3)}\left(v_{j}\right) / \partial v^{4}+g_{l}\left(u_{i}\right) \\
& \left.\partial^{6} \mathbf{C}_{n}^{(3)}\left(v_{j}\right) / \partial v^{6}\right] \\
& (n=1,2, \cdots, 6)
\end{aligned}
$$

When $i=4$, the boundary functions $\mathbf{C}_{n}^{(4)}=\mathbf{C}_{n}^{(4)}(v, t) \quad($ $n=1,2, \cdots, 6)$ are the functions of the parametric variable $v$ and the time variable $t$, and the approximate analytical solution can be obtained below with the same method as above

$$
\begin{aligned}
& \mathbf{S}^{(4)}(u, v, t)=\sum_{n=1}^{6}\left\{\sum_{l=1}^{6} \mathbf{e}_{n, l} g_{l}(u)-\sum_{m=1}^{M} \mathbf{b}_{n m}\right. \\
& {\left[m \pi f_{m}(u)-\sin m \pi u\right] \mathbf{C}_{n}^{(4)}(v, t)}
\end{aligned}
$$

where $\mathbf{e}_{n, m}(n, m=1,2, \cdots, 6)$ have three components $e_{q n, m}$ ( $q=x, y, z)$ with $e_{q n, m}=1$ for $n=m$ and $e_{q n, m}=0$ for $n \neq m$, $g_{l}(u) \quad(l=1,2, \cdots, 6)$ are the same as those in Eq. (6), $f_{m}(u)=g_{2}(u)+(-1)^{m} g_{5}(u) \quad(m=1,2, \cdots, M)$, and $\mathbf{b}_{n m}$ can be determined in the similar way to that of $i=3$.

\section{Accuracy, efficiency and applications}

In this section, we implement the developed approximate analytical solution, compare it with the corresponding accurate closed form solution to demonstrate its good accuracy and high efficiency, discuss the influence of second partial derivatives with respect to the parametric variable $u$ on the continuity at timelines, and examine constant surface blending with curvature continuity.

The approximate analytical solution and the corresponding accurate closed form solution, i. e. Equation (16) below, were implemented with $\mathrm{C}++$ and OpenGL. All the examples were run on a same laptop with $2.5 \mathrm{GHz} \mathrm{CPU}$.

4.1 Comparison between the obtained approximate analytical solution and the corresponding accurate closed form solution

First, we use a surface blending example to make a comparison between the developed approximate analytical solution and the accurate closed form solution. The comparison is used to demonstrate good accuracy and high efficiency of the developed approximate analytical solution.
This example is to create a time-dependent blending surface between two separate elliptic cylinders represented with timedependent varying primary surfaces. The top primary surface for this example is defined by

$$
\begin{aligned}
& x=a e^{t} \sin 2 \pi v \\
& y=b e^{-t} \cos 2 \pi v \\
& z=h_{1}+h_{2} u^{2}
\end{aligned}
$$

and the bottom parametric surface is represented by the equation of

$$
\begin{aligned}
& x=c e^{-t} \sin 2 \pi v \\
& y=d e^{t} \cos 2 \pi v \\
& z=-h_{3} u^{3}
\end{aligned}
$$

Deriving the first and second derivatives of Equations (13) and (14) with respect to the parametric variable $u$, setting $u=u_{1}$ in Equation (13) and its first and second partial derivatives with respect to the parametric variable $u$, and setting $u=u_{2}$ in Equation (14) and its first and second partial derivatives with respect to the parametric variable $u$, the blending boundary conditions are obtained as

$$
\begin{array}{lll}
u=0 & \\
S_{x}=a e^{t} \sin 2 \pi v & S_{y}=b e^{-t} \cos 2 \pi v & S_{z}=h_{1}+h_{2} u_{1}^{2} \\
\frac{\partial S_{x}}{\partial u}=0 & \frac{\partial S_{y}}{\partial u}=0 & \frac{\partial S_{z}}{\partial u}=-2 h_{2} u_{1} \\
\frac{\partial^{2} S_{x}}{\partial u^{2}}=0 & \frac{\partial^{2} S_{y}}{\partial u^{2}}=0 & \frac{\partial^{2} S_{z}}{\partial u^{2}}=2 h_{2} \\
u=1 & S_{y}=d e^{t} \cos 2 \pi v & S_{z}=-h_{3} u_{2}^{3} \\
S_{x}=c e^{-t} \sin 2 \pi v & \frac{\partial S_{z}}{\partial u}=-3 h_{3} u_{2}^{2} \\
\frac{\partial S_{x}}{\partial u}=0 & \frac{\partial S_{y}}{\partial u}=0 & \frac{\partial^{2} S_{z}}{\partial u^{2}}=-6 h_{3} u_{2} \\
\frac{\partial^{2} S_{x}}{\partial u^{2}}=0 & \frac{\partial^{2} S_{y}}{\partial u^{2}}=0 &
\end{array}
$$

The functions in the blending boundary constraints (15) can be divided into $\mathbf{C}_{n}^{(1)}$ and $\mathbf{C}_{n}^{(4)}(v, t)$. Using the approximate analytical solutions developed in the above section and the geometric parameters $a=1.6, b=1.0, c=0.8, d=0.6, \quad h_{1}=2.0, h_{2}=3.0$, and $h_{3}=5.0$, and setting $u=0.2$ for the top surface, $u=0.3$ for the bottom surface, the time variable to $t=0.2$, and $M$ in Eq. (12) to 10,15 , and 20 , the obtained blending surfaces generated with uniformly distributed 10,201 vertices are depicted in Figure 1.

For this example, the boundary constraints for $t=0.2$ only involve constants, and sine and cosine functions. Therefore, the closed form solution is obtainable. Using the same geometric parameters, the mathematical expressions of the corresponding closed form solution is obtained as

$$
\begin{gathered}
S_{x}(u, v)=\left[C_{x 1} e^{q_{0} u}+C_{x 2} e^{-q_{0} u}+e^{q_{1} u}\left(C_{x 3} \cos q_{2} u+C_{x 4}\right.\right. \\
\left.\left.\sin q_{2} u\right)+e^{-q_{1} u}\left(C_{x 5} \cos q_{2} u+C_{x 6} \sin q_{2} u\right)\right] \sin 2 \pi v \\
S_{y}(u, v)=\left[C_{y 1} e^{q_{0} u}+C_{y 2} e^{-q_{0} u}+e^{q_{1} u}\left(C_{y 3} \cos q_{2} u+C_{y 4}\right.\right. \\
\left.\left.\sin q_{2} u\right)+e^{-q_{1} u}\left(C_{y 5} \cos q_{2} u+C_{y 6} \sin q_{2} u\right)\right] \cos 2 \pi v \\
S_{z}(u)=\sum_{n=1}^{6} C_{z n} u^{n-1}
\end{gathered}
$$

where the constants $q_{0}, q_{1}, q_{2}, C_{x n}, C_{y n}$, and $C_{z n}$ ( $n=$ $1,2, \ldots, 6)$ are determined by the partial differential equation (3) and boundary constraints (15). 
The blending surface obtained from the accurate closed form solution is also shown in Figure 1 where CFS indicates the closed form solution. The first image of the second row of the figure shows the profile curves of the blending surface obtained from $\mathrm{M}=10,15$, and 20 of the proposed approach and the closed form solution, and the profile curves are magnified in the second image of the second row with the two innermost profile curves from $M=10$. The second row of Figure 1 shows no visible difference between profile curves from $M=15$ and $M=20$ of the proposed approach and the closed form solution.

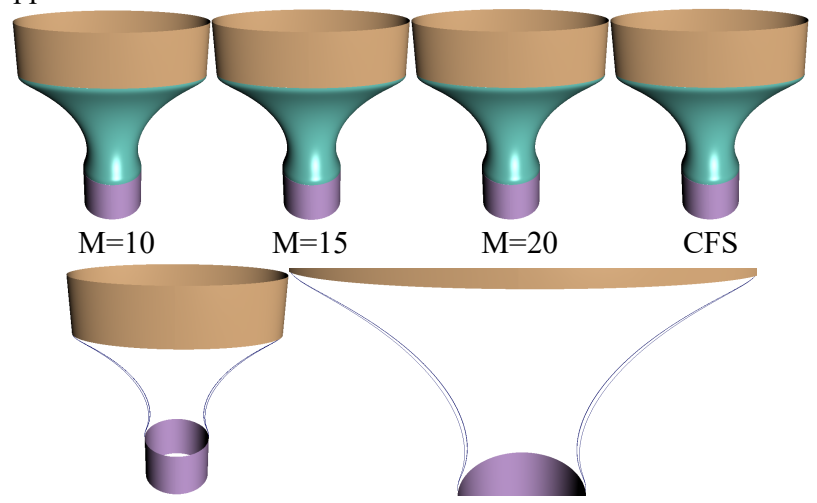

Fig. 1 Blending surfaces generated by different $M$ values.

The errors of the proposed approach against the corresponding accurate closed form solution are given in Table 1. The computational time are also given in the same table where AAE, AME, RAE, and RME indicate the absolute average error, absolute maximum error, relative average error, and relative maximum error, respectively.

The images depicted in Figure 1 and the data given in Table 1 demonstrate good accuracy and high computational efficiency of the proposed approach. When $\mathrm{M}$ increases from 10 to 20 , the relative average error of the proposed approach decrease from $7.95 \times 10^{-3}$ to $4.94 \times 10^{-6}$, and the computational time increases from 20.8 milliseconds to 75.5 milliseconds which is at the same order as the accurate closed form solution. Unlike the accurate closed form solution which is applicable to simple blending boundary constraints involving constants, sine and cosine functions, and exponential functions only, the proposed approach is applicable to various complicated blending boundary constraints.

Table 1. Accuracy and efficiency of the proposed approach

\begin{tabular}{|c|c|c|c|c|}
\hline$M$ & 10 & 15 & 20 & CFS \\
\hline AAE & $1.71 \times 10^{-2}$ & $1.23 \times 10^{-3}$ & $1.06 \times 10^{-5}$ & 0 \\
\hline AME & $4.55 \times 10^{-2}$ & $3.31 \times 10^{-3}$ & $1.30 \times 10^{-5}$ & 0 \\
\hline RAE & $7.95 \times 10^{-3}$ & $5.72 \times 10^{-4}$ & $4.94 \times 10^{-6}$ & 0 \\
\hline RME & $2.11 \times 10^{-2}$ & $1.54 \times 10^{-3}$ & $1.07 \times 10^{-5}$ & 0 \\
\hline $\begin{array}{c}\text { Time } \\
(\mathrm{ms})\end{array}$ & 20.8 & 43.2 & 75.5 & 17.8 \\
\hline
\end{tabular}

With the developed method and setting the time variable $t=0$ , $t=0.1, t=0.2$, and $t=0.3$, the blending surfaces at these time instants are obtained and depicted in Figure 2 where the first row is from the front view and the second row is from the side view.

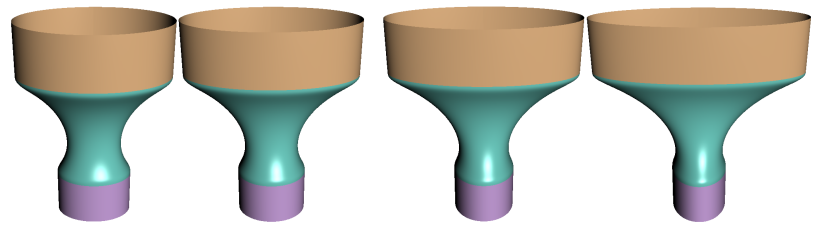

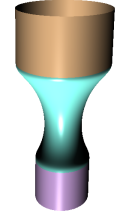

$t=0.0$

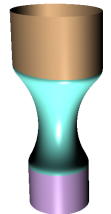

$t=0.1$

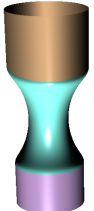

$t=0.2$

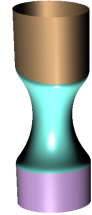

$t=0.3$
Fig. 2 Blending surfaces at different time instants.

The images shown in Figure 2 indicates that at different time instants, the proposed approach always creates blending surfaces of curvature continuity to smoothly connect primary surfaces together.

\subsection{Effects of second partial derivatives}

Unlike surface blending with tangential continuity presented in [5] which only maintains the continuities of the position functions and first partial derivatives at trimlines, the surface blending with curvature continuity proposed in this paper further introduces second partial derivatives at trimlines to achieve higher continuity. In this subsection, we will demonstrate how second partial derivatives at trimlines affect the continuity between the blending surface and primary surfaces.

Setting $t=0.05$ and keeping all the position functions, all the first partial derivatives, and the second partial derivatives of the surface functions $x$ and $y$ for both the blending surface and primary surfaces at timelines same as those given in Eq. (15), the following three different cases of the second partial derivatives of the surface function $z$ shown in Table 2 are considered where TPS, BS and BPS stand for the top primary surface, blending surface, and bottom primary surface, respectively. For all the three cases, the top primary surface has the second partial derivative $-2 h_{2}$ at the trimline $u=0$ and the bottom primary surface has the second partial derivative $-1.8 h_{3}$ at the trimline $u=1$.

Table 2. Second partial derivatives of the surface function $z$ with respect to $u$ at trimlines

\begin{tabular}{|c|l|l|l|l|l|}
\hline & $\begin{array}{l}\text { TPS } \\
u=0.2\end{array}$ & $\begin{array}{l}\text { BS } \\
u=0\end{array}$ & $\begin{array}{l}\text { BPS } \\
u=0\end{array}$ & $\begin{array}{l}\text { BS } \\
u=0.3\end{array}$ & Figure \\
\hline \multirow{2}{*}{$\frac{\partial^{2} z}{\partial u^{2}}$} & $-2 h_{2}$ & $-2 h_{2}$ & $-1.8 h_{3}$ & $-1.8 h_{2}$ & $3(\mathrm{a})$ \\
\cline { 2 - 6 } & $-2 h_{2}$ & $-10 h_{2}$ & $-1.8 h_{3}$ & $-5.4 h_{2}$ & $3(\mathrm{~b})$ \\
\cline { 2 - 6 } & $-2 h_{2}$ & $-20 h_{2}$ & $-1.8 h_{3}$ & $-10.8 h_{2}$ & $3(\mathrm{c})$ \\
\hline
\end{tabular}

Case 1: The blending surface and primary surfaces have the same second partial derivatives at both trimlines.

Case 2: The blending surface has the second partial derivative $-10 h_{2}$ at $u=0$ and $-5.4 h_{3}$ at $u=1$.

Case 3: The blending surface has the second partial derivative $-20 h_{2}$ at $u=0$ and $-10.8 h_{3}$ at $u=1$.
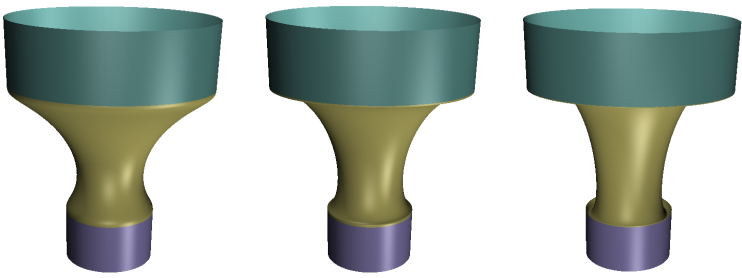


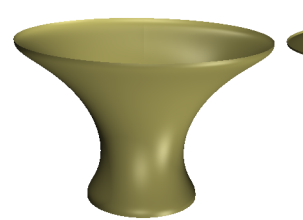

(a)

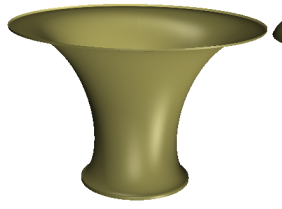

(b)

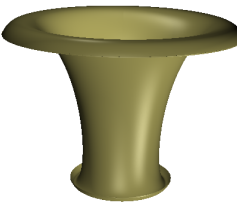

(c)
Fig. 3 Effects of second partial derivatives

The obtained results for the three cases are shown in Figures 3 where the surfaces in the bottom row show the blending shapes only. They are used to demonstrate how the second partial derivatives affect the continuity at the trimlines.

Comparing these images, we can conclude: 1) when the blending surface and primary surfaces have the same second partial derivatives at the trimlines, good continuity between the blending surface and the primary surfaces is obtained as shown in Figure $3(\mathrm{a})$; 2) different second partial derivatives between the blending surface and primary surfaces will cause discontinuity at the trimlines as indicted in Figures 3(b) and 3(c); 3) the larger is the difference of the second partial derivatives between the blending surface and primary surfaces, the more serious is the discontinuity as demonstrated in Figure 3(c).

\subsection{Blending of constant parametric surfaces}

Finally, we investigate how the developed approach can be used to blend constant parametric surfaces with curvature continuity. This example is to blend two perpendicular planes which is a very common engineering problem. The blending boundary constraints for this surface belnding can be taken to be

$$
\begin{aligned}
& u=0 \quad S_{x}=0 \quad S_{y}=h_{0} \quad S_{z}=p v \\
& \frac{\partial S_{x}}{\partial u}=0 \quad \frac{\partial S_{y}}{\partial u}=-h_{1} \quad \frac{\partial S_{z}}{\partial u}=0 \\
& \frac{\partial^{2} S_{x}}{\partial u^{2}}=0 \quad \frac{\partial^{2} S_{y}}{\partial u^{2}}=-h_{1} \quad \frac{\partial^{2} S_{z}}{\partial u^{2}}=0 \\
& u=1 \quad S_{x}=s_{0} \quad S_{y}=0 \quad S_{z}=p v \\
& \frac{\partial S_{x}}{\partial u}=s_{1} \quad \frac{\partial S_{y}}{\partial u}=0 \quad \frac{\partial S_{z}}{\partial u}=0 \\
& \frac{\partial^{2} S_{x}}{\partial u^{2}}=0 \quad \frac{\partial^{2} S_{y}}{\partial u^{2}}=0 \quad \frac{\partial^{2} S_{z}}{\partial u^{2}}=0
\end{aligned}
$$

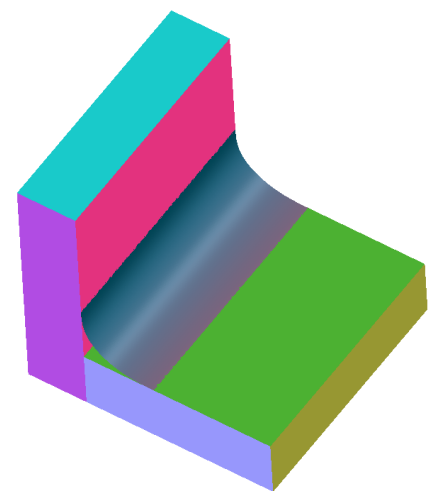

Fig. 4 Blending between perpendicular planes

The geometric parameters in Equation (17) are taken to be $h_{0}=0.5, p=2, h_{1}=1, s_{0}=0.6$, and $s_{1}=0.9$. The developed approximate analytical solution is used to create the blending surface which is shown in Figure 4. It is clear that the proposed approach is effective in blending constant parametric surfaces with curvature continuity.
The second example is to blend the frustum of a declined circular cone and a declined plane at a specified circle with curvature continuity. The top parametric surface can be described by the following equations

$$
\begin{aligned}
& x=r u^{2} \cos v \\
& y=r u^{2} \sin v \\
& z=h_{0}+h_{1} u+h \cos v
\end{aligned}
$$

and the bottom parametric surface is represented by the equation of

$$
\begin{aligned}
& x=R u \cos \alpha \cos v \\
& y=R u \sin v \\
& z=R u \sin \alpha \cos v
\end{aligned}
$$

If the top surface is to be connected to the blending surface at $u=u_{0}$, and the bottom surface is to be connected to the blending surface at $u=u_{1}$, the blending boundary constraints can be formulated as

$$
\begin{aligned}
& u=0 \\
& S_{x}=r u_{0}^{2} \cos v \quad S_{y}=r u_{0}^{2} \sin v \quad S_{z}=h_{0}+h_{1} u_{0}+h \cos v \\
& \frac{\partial S_{x}}{\partial u}=-2 r u_{0} \cos v \quad \frac{\partial S_{y}}{\partial u}=-2 r u_{0} \sin v \quad \frac{\partial S_{z}}{\partial u}=-h_{1} \\
& \frac{\partial^{2} S_{x}}{\partial u^{2}}=2 r \cos v \quad \frac{\partial^{2} S_{y}}{\partial u^{2}}=2 r \sin v \quad \frac{\partial^{2} S_{z}}{\partial u^{2}}=0 \\
& u=1 \\
& S_{x}=R u_{1} \cos \alpha \cos v \quad S_{y}=R u_{1} \sin v \quad S_{z}=R u_{1} \sin \alpha \cos v \\
& \frac{\partial S_{x}}{\partial u}=R \cos \alpha \cos v \quad \frac{\partial S_{y}}{\partial u}=R \sin v \quad \frac{\partial S_{z}}{\partial u}=R \sin \alpha \cos v \\
& \frac{\partial^{2} S_{x}}{\partial u^{2}}=0 \quad \frac{\partial^{2} S_{y}}{\partial u^{2}}=0
\end{aligned}
$$
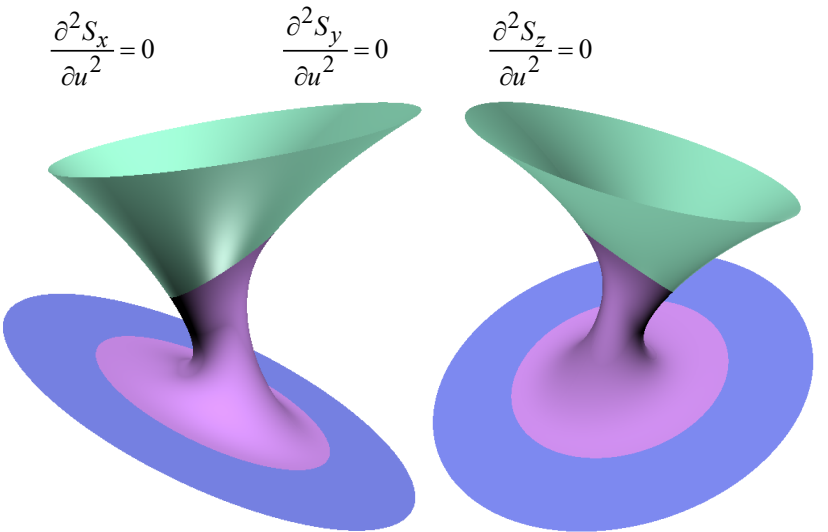

(a) (b)
Fig. 5 Blending between the frustum of a declined circular cone and a declined plane at a specified circle

Taking $u_{0}=0.6$ and $u_{1}=0.8$ in Eq. (20), and setting the geometric parameters to: $r=1.8, R=2, h_{0}=h=0.5, h_{1}=3$, and $\alpha=-20^{\circ}$, we obtain the blending surface and depict it in Figure 5 where the two images shown in Figure 5(a) and Figure 5(b) are from different view angles.

\section{Conclusions}

In this paper, we have developed a new surface blending method to create a blending surface of curvature continuity between two constant and varying parametric surfaces.

In order to develop this method, we have formulated the mathematical model consisting of a vector-valued sixth-order partial differential equation and blending boundary constraints, and obtained an approximate analytical solution to achieve surface blending of curvature continuity easily and efficiently. The 
comparison between the approximate analytical solution and the corresponding accurate closed form solution demonstrates that the developed approximate analytical solution has good accuracy and high computational efficiency.

We also investigated how the second partial derivatives affect the continuity at trimlines. It was found that the same second partial derivatives between the blending and primary surfaces guarantee good continuity at the trimlines, but different second partial derivatives between the blending and primary surfaces cause discontinuity at the trimlines.

Finally, we employed the developed approximate analytical solution to blend constant surfaces whose positions and shapes do not change with the time. The application demonstrates the developed approximate analytical solution is effective in dealing with blending of both constant and varying parametric surfaces with curvature continuity.

\section{References}

1. Hartmann, E.: Implicit $G^{n}$-blending of vertices. Comput. Aided Geom. Des. 18, 267-285 (2001)

2. Song, Q., Wang, J.: Generating $G^{n}$ parametric blending surfaces based on partial reparameterization of base surfaces. Comput. Aided Des. 39, 953-963 (2007)

3. Kiciak, P.: Bicubic B-spline blending patches with optimized shape. Comput. Aided Des. 43, 133-144 (2011)

4. Vida, J., Martin, R.R., Varady, T.: A survey of blending methods that use parametric surfaces. Comput. Aided Des. 26(5), 341-365 (1994)

5. You, L.H., Ugail, H., Zhang, J.J.: Controllable C1 continuous blending of time-dependent parametric surfaces. Vis. Comput. 28, 573-583 (2012).

6. Rossignac, J.R., Requicha, A.A.G.: Constant-radius blending in solid modeling. CIME, Comput. Mech. Eng. 3(1), 65-73 (1984)

7. Farouki, R.A.M., Sverrisson, R.: Approximation of rollingball blends for free-form parametric surfaces. Comput. Aided Des. 28(11), 871-878 (1996)

8. Kós, G., Martin, R.R., Vrady, T.: Methods to recover constant radius rolling ball blends in reverse engineering. Comput. Aided Geom. Des. 17, 127-160 (2000)

9. Dahl, H.E.I., Krasauskas, R.: Rational fixed radius rolling ball blends between natural quadrics. Comput. Aided Geom. Des. 29, 691-706 (2012)

10. Chuang, J.H., Hwang, W.C.: Variable-radius blending by constrained spine generation. Vis. Comput. 13, 316-329 (1997)

11. Chuang, J.-H., Lien, P.-L.: One and two-parameter blending for parametric surfaces. J. Inf. Sci. Eng. 14, 461-477 (1998)

12. Lukács, G.: Differential geometry of G1 variable-radius rolling ball blend surfaces. Comput. Aided Geom. Des. 15, 585-613 (1998)

13. Allen, G., Dutta, D.: Cyclides in pure blending II. Comput. Aided Geom. Des. 14, 77-102 (1997)

14. Shene, C.-K.: Blending two cones with Dupin cyclides. Comput. Aided Geom. Des. 15, 643-673 (1997)

15. Roach, P.A., Martin, R.R.: Production of blends and fairings by Fourier methods. SPIE: Curves and Surfaces in Computer Vision and Graphics III, 1830: 162-173 (1992)

16. Kim, K., Elber, G.: A symbolic approach to freeform parametric surface blends. The Journal of Visualisation and Computer Animation 8, 69-80 (1996)

17. Schichtel, M.: $G 2$ blend surfaces and filling of N-sided holes. IEEE Computer Graph. Appl. 13(9), 68-73 (1993)
18. Hsu, K.L., Tsay, D.M.: Corner blending of free-form N-sided holes. IEEE Computer Graph. Appl. 18(1), $72-78$ (1998)

19. Piegl, L.A., Tiller, W.: Filling $n$-sided regions with NURBS patches. Vis. Computer 15(2), 77-89 (1999)

20. Hwang, W.C., Chuang, J.H.: $N$-sided hole filling and vertex blending using subdivision surfaces. J. Inf. Sci. Eng. 19, 857879 (2003)

21. Yang, Y.-J., Yong, J.-H., Zhang, H., Paul, J.-C., Sun, J.-G.: A rational extension of Piegl's method for filling $n$-sided holes. Comput. Aided Des. 38(11), 1166-1178 (2006)

22. Shi, K.-L., Yong, J.-H., Sun, J.-G.: Filling $n$-sided regions with $G^{1}$ triangular Coons B-spline patches. Vis. Computer 26, 791-80 (2010)

23. Zhou, P., Qian, W.-H.: A vertex-first parametric algorithm for polyhedron blending. Comput. Aided Des. 41, 812-824 (2009)

24. Zhou, P.: Polyhedral vertex blending with setbacks using rational S-patches. Comput. Aided Geom. Des. 27, 233-244 (2010)

25. Krasauskas, R.: Branching blend of natural quadrics based on surfaces with rational offsets. Comput. Aided Geom. Des. 25, 332-341 (2008)

26. Bloor, M.I.G., Wilson, M.J.: Generating blend surfaces using partial differential equations. Comput. Aided Des. 21(3), 165171 (1989)

27. Brown, J.M., Bloor, M.I.G., Bloor, M.S., Wilson, M.J.: The accuracy of B-spline finite element approximations to PDE surfaces. Comput. Methods Appl. Mech. Eng. 158(3-4), 221234 (1998)

28. Li, Z.C., Chang, C.-S.: Boundary penalty finite element methods for blending surfaces. III. Superconvergence and stability and examples. J. Comput. Appl. Math. 110, 241-270 (1999)

29. You, L.H., Zhang, J.J., Comninos, P.: Blending surface generation using a fast and accurate analytical solution of a fourth order PDE with three shape control parameters. Vis. Comput. 20, 199-214 (2004)

30. You, L.H., Comninos, P., Zhang, J.J.: PDE blending surfaces with C2 continuity. Comput. Graph. 28(6), 895-906 (2004) 